\title{
Seiberg's Duality from Monodromy of Conifold Singularity
}

\author{
Kei Ito \\ Department of Electrical and Computer Engineering \\ Nagoya Institute of Technology \\ Gokiso, Showa-ku, Nagoya 466-8555, Japan \\ E-mail: keiito@eken.phys.nagoya-u.ac.jp or ito@ks.kyy.nitech.ac.jp
}

\begin{abstract}
Duality between $\mathrm{N}=1$ supersymmetric gauge theories(Seiberg's duality) is geometrized, in the framework of AdS/CFT correspondences. It is shown that Seiberg's duality corresponds to monodromy of wrapped D5 branes on the homology cycles of a generalized conifold where D3 branes are located. The celebrated $\tilde{N}_{c}=N_{f}-N_{c}, \tilde{N}_{f}=N_{f}$ rule is reproduced and a braid group structure in a sequence of dualities, is found.
\end{abstract}

PACS; 11.25.Sq; 11.30.Pb

Keywords; Seiberg's duality, AdS/CFT correspondence, monodromy, conifold, singularity 
Recently, there has been great progress in our understanding of the behavior of four dimensional supersymmetric gauge theories, in the strong coupling regime. In particular, Maldacena[1] conjectured that $\mathrm{N}=4$ supersymmetric gauge theory (conformal field theory) in four dimensions, is dual to type IIB string theory on a product of five dimensional anti-de $\operatorname{Sitter}\left(A d S_{5}\right)$ space and five dimensional sphere $\left(S^{5}\right)$. (AdS/CFT correspondence). Subsequently, Klebanov and Witten[2] generalized AdS/CFT correspondence to a supersymmetric gauge theory with less supersymmetry, i. e. $N=1$. There they showed that a certain $\mathrm{N}=1$ supersymmetric gauge theory, which arises as a field theory of parallel threebranes near a conifold singularity of a six dimensional Calabi-Yau space $Y_{6}$ corresponds to type IIB string theory on $A d S_{5} \times X_{5}$, with $X_{5}$ being a five dimensional Einstein manifold, $(S U(2) \times S U(2)) / U(1)$. (See also ref.[3]) Gubser et al.[4] and Lopez[5] generalized this correspondences to a class of more general conifolds $Y_{6}$ classified according to simply-laced Lie algebras of ADE type, which is a cone over $X_{5}$ obtained by blowing up an orbifold singularity of $S^{5} / \Gamma$, with $\Gamma$ being diescrete group classified according to the same type of the simply-laced Lie algebra. These correspondences suggest that the strong coupling behavior of $\mathrm{N}=1$ supersymmetric gauge theories in four dimensions are encoded in geometry of corresponding conifold singularity. There are dualities between $\mathrm{N}=1$ supersymmetric gauge theories(Seiberg's dualities) [6] where the dynamics in the strong coupling regime are responsible. Therefore, it is tempting to conjecture that Seiberg's duality can be geometrized to an operation on the conifold singularity by means of AdS/CFT correspondences. This is the problem which we address in this paper. We will find that Seiberg's duality corresponds to a geometrical operation on the conifold which is a monodromy of homology 2-cycles of the conifold called Picard-Lefschetz monodromy. If there are D5 branes wrapped on homology 2-cycles, in addition to a stack of D3 branes, we will have a non-trivial Seiberg's duality and obtain the celebrated rule, $\tilde{N}_{c}=N_{f}-N_{c}, \tilde{N}_{f}=N_{f}$. Moreover, we will find a braid group structure in a sequence of Seiberg's dualities by virtue of this geometrization.

Following references [4],[5],we review $\mathrm{N}=1$ supersymmetric field theories which arise from D3 branes on generalized conifold of ADE-type. Consider N D3 branes located at an orbifold singularity $\mathbf{C}^{\mathbf{2}} / \boldsymbol{\Gamma} \times \mathbf{C}$, where $\Gamma$ is a discrete subgroup of $\mathrm{SU}(2)$ classified by simply-laced Lie algebra ADE. The effective field theory on D3 branes is a four-dimensional $\mathrm{N}=2$ supersymmetric gauge theory with a product gauge group, $\prod_{i=0}^{k} S U\left(N n_{i}\right)$, where, $\mathrm{k}$ is the rank of the Lie algebra corresponding to the discrete group $\Gamma$, and $n_{i}=\operatorname{dim} \mathbf{r}_{\mathbf{i}}$ 
with $\mathbf{r}_{\mathbf{i}}$ being the $\mathrm{i}$-th representation of $\Gamma$. In the corresponding simplylaced Lie algebra, $n_{i}$ is identical to Dynkin label of the node of extended Dynkin diagram. The matters which arise are $a_{i j}$ copies of hypermultiplets in the bi-fundamental represenations, $\left(n_{i} N, \overline{n_{j} N}\right)$ where $a_{i j}$ comes from the decomposition $\mathbf{C}^{\mathbf{2}} \otimes \mathbf{r}_{\mathbf{i}}=\oplus_{\mathbf{j}} \mathbf{C}^{\mathbf{a}_{\mathbf{i j}}} \mathbf{r}_{\mathbf{j}}$. The orbifold $\mathbf{C}^{\mathbf{2}} / \boldsymbol{\Gamma}$ can be represented as an algebraic variety, by a hypersurface defined by the equation $f_{\Gamma}=0$ in $\mathbf{C}^{\mathbf{3}}$ parametrized by three complex numbers $x, y$ and $z$.

$$
\begin{array}{lrlrl}
f_{\Gamma}=x^{k+1}+y^{2}+z^{2}, & & \left(\text { for } A_{k}\right) \\
f_{\Gamma}=x^{k-1}+x y^{2}+z^{2}, & & \left(\text { for } D_{k}\right) \\
f_{\Gamma}=x^{4}+y^{3}+z^{2}, & & \left(\text { for } E_{6}\right) \\
f_{\Gamma}=x^{3} y+y^{3}+z^{2}, & & \left(\text { for } E_{7}\right) \\
f_{\Gamma}=x^{5}+y^{3}+z^{2} . & & \left(\text { for } E_{8}\right)
\end{array}
$$

The equation $f_{\Gamma}=0$ is invariant under the $\mathbf{C}^{*}$ action, $x \rightarrow \lambda^{\alpha} x, y \rightarrow \lambda^{\beta} y, z \rightarrow$ $\lambda^{\gamma} z$ where, $\alpha=1, \beta=\frac{k+1}{2}, \gamma=\frac{k+1}{2}$, in $A_{k}$ case,for instance, since $f_{\Gamma}$ scales as $\lambda^{h} f_{\Gamma}$, with $h=k+1$, under this action.

An $N=1$ supersymmetric theory is obtained if the orbifold singularity is resolved to become a non-compact Calabi- Yau manifold $Y_{\Gamma}$ with a conifoldlike singularity. The orbifold singularity is deformed by parameters t's, which is then fibered on a complex plane parametrized by $\phi$. The defining equation of this "generalized conifold" is then given by;

$$
F_{\Gamma}(\phi, x, y, z)=\phi^{h} f_{\Gamma}\left(\frac{x}{\phi^{\alpha}}, \frac{y}{\phi^{\beta}} ; t\right)+z^{2}=0 .
$$

For instance, the holomorphic function $\mathrm{F}$ for $\Gamma=A_{k}$ is;

$$
\begin{aligned}
F_{\Gamma}(\phi, x, y, z) & =x^{k+1}+\phi^{2} t_{2} x^{k-1}+\ldots+\phi^{k} t_{k} x+y^{2}+z^{2} \\
& =\prod_{i=1}^{k+1}\left(x-\xi_{i} \phi\right)+y^{2}+z^{2}
\end{aligned}
$$

Let us find the effective field theory on the branes located at this generalized conifold. The T-dual brane configuration pictures[7],[8],[9] are more convenient for this purpose. Let N D3 branes (world-volume in 0123 directions) be located at the orbifold singularity of ALE space ( $A_{k}$-type, for simplicity)with coordinates 6789. Perform T-duality along 6ith direction, corresponding to the U(1) isometry of the ALE space.[7] Then we obtain type II A string with 
$\mathrm{k}+1$ NS 5 branes (world-volume in 012345 directions). D3 branes are transformed into D4 branes (01236). The effective field theory on the D4 branes, is $\mathrm{N}=2$ supersymmetric gauge theory with the gauge group $S U(N)^{k+1}$. If, N D3 branes are located at a generalized conifold,[8],[9] instead of ALE[7], we obtain, $\mathrm{k}+1$ NS 5 branes rotated in the complex surface spanned by, $x=x^{8}+i x^{9}$ and $\phi=x^{4}+i x^{5}$. In this case, scalar fields in adjoint representations of $S U(N)^{k+1}$ become massive and supersymmetry is broken from $\mathrm{N}=2$ to $\mathrm{N}=1$. The spacing of $\mathrm{i}$-th and (i+1)-th NS 5 branes in 6 -th direction, is related to the coupling constant $g_{i}$ of the i-th factor $S U(N)_{i}$ of the product gauge group, by;

$$
\frac{x^{6}\left(N S_{i+1}\right)-x^{6}\left(N S_{i}\right)}{g_{s}}=\frac{1}{g_{i}^{2}}
$$

where $g_{s}$ is the string coupling constant. The spacing in 7-th direction, on the other hand, is related to the coefficient of Fayet-Iliopoulos(FI) term $\zeta_{i}$ of the i-th gauge factor through,

$$
x^{7}\left(N S_{i+1}\right)-x^{7}\left(N S_{i}\right)=\zeta_{i}
$$

Seiberg's duality with respect to the i-th gauge group factor of the product group corresponds to an interchange of $\mathrm{i}$-th and (i+1)-th NS 5 branes by the following procedure.[8],[10],[11] The $\mathrm{i}$-th and the (i+1)-th NS 5 branes come close together in 6 -th direction until $x^{6}\left(N S_{i+1}\right)-x^{6}\left(N S_{i}\right)$ reaches a small real value $\delta$. In the field theory side, the coupling costant $g_{i}$ becomes larger and larger until $\frac{1}{g_{i}^{2}}$ reaches $\frac{\delta}{g_{s}}$. Then the FI term $\zeta_{i}$ turns on in order to avoid passing through the infinite coupling constant point $g_{i}=\infty$. Then $g_{i}$ and $\zeta_{i}$ vary as;

$$
\frac{g_{s}}{g_{i}^{2}}+i \zeta_{i}=\delta e^{\pi i t}
$$

where, $\mathrm{t}$ runs from 0 to 1 . At $\mathrm{t}=1$, FI term is turned off and the order of two NS5 branes are interchanged in 6-th direction, since 6-th and 7-th coordinates of NS5 branes change as;

$$
\left[x^{6}\left(N S_{i+1}\right)-x^{6}\left(N S_{i}\right)\right]+i\left[x^{7}\left(N S_{i+1}\right)-x^{7}\left(N S_{i}\right)\right]=\delta e^{\pi i t} .
$$

Finally the separation of NS5 branes becomes larger and larger until the gauge coupling constant comes back to the original value.

Now let us come back to the original picture, i.e. type II B string with N D3 branes at the conifold, by T-duality transformation. Then, 
$x^{6}\left(N S_{i+1}\right)-x^{6}\left(N S_{i}\right)$ and $x^{7}\left(N S_{i+1}\right)-x^{7}\left(N S_{i}\right)$ are real and imaginary part of complexified kähler class $[\mathrm{k}]$ of homology 2-cycles of the conifold. We find that the procedure corresponding to Seiberg's duality; two NS5 branes come clos together, being interchanged, and go away, is a geometrical operation on the singularity of the conifold, which is called "Picard-Lefschetz monodromy".

Picard-Lefschetz monodromy of a singularity is defined as follows. See the book by Arnold et al.[12] for detail. This monodromy plays also a crucial role in two-dimensional conformal field theory[13]. Consider generalized conifold of $A_{k}$-type defined by the equation;

$$
F_{\Gamma}(\phi, x, y, z)=0
$$

The level manifold of level $\lambda \in \mathbf{C}$, is defined by the equation, $F_{\Gamma}(\phi, x, y, z)=$ $\lambda$. The critical points of the holomorphic function $F_{\Gamma}$ is defined by,

$$
\frac{\partial F_{\Gamma}}{\partial x}=0, \frac{\partial F_{\Gamma}}{\partial y}=0, \frac{\partial F_{\Gamma}}{\partial z}=0 .
$$

This is a k-th order algebraic equation which has k solutions; $x=X_{i}(i=$ $1,2, \ldots, k), y=0, z=0$. The values of the holomorphic function $\mathrm{F}$ at critical points $x=X_{i}$, are called critical values $\lambda_{i}(i=1, \ldots, k)$. For example, for $\Gamma=A_{k}$

$$
\lambda_{i}=\prod_{j=1}^{k+1}\left(X_{i}-\xi_{j} \phi\right) .
$$

If the level value $\lambda$ starts from a non-critical value $\lambda_{0}$, goes around one of the critical values $\lambda_{i}$ and comes back to the the original value $\lambda_{0}$, the level manifold comes back to the original one but the homology cycles (vanishing cycles) are reshuffled. The holomorphic function F is expanded around the critical values;

$$
F_{\Gamma}=\lambda_{i}+w_{1}^{2}+w_{2}^{2}+w_{3}^{2} .
$$

Then, i-th vanishing cycle $\Delta_{i}$ is[12]

$$
\Delta_{i}=\sqrt{\lambda-\lambda_{i}} S^{2},
$$

where, $\sqrt{\lambda-\lambda_{i}}$ is the complexified kahler class and $S^{2}$ is a unit two-sphere.

$$
S^{2}=\left\{\left(w_{1}, w_{2}, w_{3}\right) \mid \sum_{j=1}^{3} w_{j}^{2}=1, I m w_{j}=0\right\}
$$


The Picard-Lefschetz monodromy is the operation where the level value $\lambda$ goes around a critical value $\lambda_{i}$.

$$
\lambda-\lambda_{i}=\delta^{2} e^{2 \pi i t}, \text { for small } \delta, t \in[0,1] .
$$

This implies that

$$
\sqrt{\lambda-\lambda_{i}}=\delta e^{\pi i t}
$$

Since $\sqrt{\lambda-\lambda_{i}}$ is the complex kahler class $[\mathrm{k}]$ of the $\mathrm{i}$-th vanishing cycle.

$$
\sqrt{\lambda-\lambda_{i}}=[k] \text {. }
$$

the change of complex kahler class $[\mathrm{k}]$ under the Picard-Lefschetz monodromy is the same as that under the interchange of the $\mathrm{i}$-th and $(\mathrm{i}+1)$-th NS 5branes in the T-dual picture, which corresponds to Seiberg's duality. Thus we find that Seiberg's duality corresponds to Picard-Lefschetz monodromy of vanishing cycles of the corresponding conifold.

The gauge theory arising from N D3 branes at generalized conifold is self-dual under the Seiberg's duality transformation. This implies that the number of colors of the dual theory are the same as those of the original theory. A non-trivial Seiberg's duality where the dual gauge group is different from the original one is realized when D5 branes are wrapped on the vanishing cycles (homology cycles) of the conifold. The wrapped D5 brane[14] has D3 brane charge which is the same as the wrapping number. Therefore, if a D5 brane is wrapped on th i-th vanishing cycle $\Delta_{i}$, with wrapping number $M_{i}$, the effective D3 brane number is changed from $\mathrm{N}$ to $N+M_{i}$. Then the i-th gauge group factor is changed from $S U(N)_{i}$ to $S U\left(N+M_{i}\right)_{i}$. Under the Picard-Lefschetz monodromy transformation, the vanishing cycles are reshuffled, and wrapping numbers of D5 branes are changed accordingly. In the field theory side, this implies that the numberof colors is changed under Seiberg's duality transformation.

Let us consider a simple example; the generalized conifold of $A_{3}$ type. $\mathrm{N}$ D3 branes on this conifold give $\mathrm{N}=1$ supersymmetric gauge theory with the gauge group $G=S U(N)_{0} \times S U(N)_{1} \times S U(N)_{2} \times S U(N)_{3}$. Each gauge group factor corresponds to the node of extended Dynkin diagram of the Lie algebra $A_{3}$. The generalized conifold of $A_{3}$ type has three vanishing cycles (homology 2-cycles) $\Delta_{1}, \Delta_{2}$ and $\Delta_{3}$, corresponding to $S U(N)_{1}, S U(N)_{2}$ and $S U(N)_{3}$. If D5 branes wrap around $\Delta_{1}, \Delta_{2}$ and $\Delta_{3}$, with wrapping numbers $M_{1}, M_{2}$ and $M_{3}$, the gauge group is,

$$
G=S U(N)_{0} \times S U\left(N+M_{1}\right)_{1} \times S U\left(N+M_{2}\right)_{2} \times S U\left(N+M_{3}\right)_{3}
$$


There are three Picard-Lefschetz monodromy transformations $h_{1}, h_{2}$ and $h_{3}$. The transformation rules of vanishing cycles $\Delta_{i},(i=1,2,3)$ under $h_{i},(i=$ $1,2,3)$ are calculated by use of Picard-Lefschetz formula[12].

$$
h_{i}(a)=a+\left(a \circ \Delta_{i}\right) \Delta_{i}
$$

where intersections of two vanishing cycles $\Delta_{i}$ and $\Delta_{j}$ are represented by the intersection matrix $S_{i j}=\Delta_{i} \circ \Delta_{j}=-A_{i j}$ where $A_{i j}$ is the Cartan matrix of $A_{3}$. Hence,

$$
S_{i j}=\left(\begin{array}{rrr}
-2 & 1 & 0 \\
1 & -2 & 1 \\
0 & 1 & -2
\end{array}\right)
$$

The action of $h_{i}(i=1,2,3)$ on $\Delta_{j}(j=1,2,3)$ can be represented by the monodromy matrix defined by the relation;

$$
h_{i}\left(\Delta_{j}\right)=\left(h_{i}\right)_{j k} \Delta_{k}
$$

Then,

$$
\begin{aligned}
h_{1} & =\left(\begin{array}{rrr}
-1 & 0 & 0 \\
1 & 1 & 0 \\
0 & 0 & 1
\end{array}\right) \\
h_{2} & =\left(\begin{array}{rrr}
1 & 1 & 0 \\
0 & -1 & 0 \\
0 & 1 & 1
\end{array}\right) \\
h_{3} & =\left(\begin{array}{rrr}
1 & 0 & 0 \\
0 & 1 & 1 \\
0 & 0 & -1
\end{array}\right)
\end{aligned}
$$

Now Seiberg's duality transformation performed on i-th gauge group factor $S U\left(N+M_{i}\right)_{i}$ corresponds to i-th Picard-Lefschetz monodromy transformation, represented by i-th monodromy matrix $h_{i}$. By this matrix, the vanishing cycles are transformed as;

$$
\Delta_{j}^{\prime}=\left(h_{i}\right)_{j k} \Delta_{k}
$$

Then the wrapping numbers of D5 branes are changed accordingly;

$$
M_{j} \Delta_{j} \rightarrow M_{j} \Delta_{j}^{\prime}=M_{j}\left(h_{i}\right)_{j k} \Delta_{k}=M_{k}^{\prime} \Delta_{k}
$$


Hence;

$$
M_{k}^{\prime}=M_{j}\left(h_{i}\right)_{j k}
$$

If Seiberg's duality transformation is performed on the gauge group factor $S U\left(N+M_{1}\right)_{1}$, the corresponding monodromy matrix is $h_{1}$. Hence,

$$
M_{1}^{\prime}=M_{j}\left(h_{1}\right)_{j 1}=M_{2}-M_{1}, M_{2}^{\prime}=M_{j}\left(h_{1}\right)_{j 2}=M_{2}, M_{3}^{\prime}=M_{j}\left(h_{1}\right)_{j 3}=M_{3} .
$$

and the dual group turns out to be;

$$
\tilde{G}=S U(N)_{0} \times S U\left(N+M_{2}-M_{1}\right)_{1} \times S U\left(N+M_{2}\right)_{2} \times S U\left(N+M_{3}\right)_{3} .
$$

Under this duality transformation, coupling constant of the gauge group factor with the suffix 1, becomes large, wich should then be regaded as the color group. therefore, the numbers of colors and flavors of the original theory are, $N_{c}=N+M_{1}$ and $N_{f}=2 N+M_{2}$, respectively,and those of the dual theory are $\tilde{N}_{c}=N+M-2-M_{1}$ and $\tilde{N}_{f}=2 N+M_{2}$, respectively.

Note that the rule, $\tilde{N}_{c}=N_{f}-N_{c}, \tilde{N}_{f}=N_{f}$ is satisfied in this example.

Seiberg's dual on group factor with the suffices 2 and 3 can be taken in the same fashion, and the above rule is satisfied also in these cases.

A general expression for the dual gauge theory can now be written. Let the $\mathrm{N}=1$ supersymmetric gauge theory with the gauge group $G=S U(N)_{0} \times$ $S U\left(n_{1} N+M_{1}\right)_{1} \times \ldots \times S U\left(n_{k} N+M_{k}\right)_{k}$ be the field theory which arises from N D3 branes on the conifold given by a below up of $\mathbf{C}^{\mathbf{2}} / \mathbf{\Gamma} \times \mathbf{C}$, with $\Gamma$ being a discrete subgroup corresponding to a rank $\mathrm{k}$ simply-laced Lie algebra, with Dynkin labels $n_{i}$, and D5 branes wrap around $\mathrm{i}-\mathrm{th}(\mathrm{i}=1, \ldots, \mathrm{k})$ homology 2cycles with wrapping number $M_{i}$. The matters arise as hypermultiplets in the bi-fundamental representations $\left(n_{i} N+M_{i}, \overline{n_{j} N+M_{j}}\right)$, if $\mathrm{i}$-th and $\mathrm{j}$-th gauge group factors are "adjacent" in the the sense that the nodes are connected by spokes in the corresponding extended Dynkin diagram. Then the dual theory is determined as follows. The intersection matrix of homology 2-cycles of the conifold is;

$$
S_{i j}=\Delta_{i} \circ \Delta_{j}=-A_{i j}
$$

where $A_{i j}$ is the Cartan matrix of the Lie algebra. Then the i-th monodromy matrix $h_{i}$ can be calculated by Picard-Lefschetz theorem;

$$
h_{i}(a)=a+\left(a \circ \Delta_{i}\right) \Delta_{i}
$$


Now if we perform Seiberg's duality transformation on i-th gauge group factor, the homology 2-cycles $\Delta_{i}$ are transformed by i-th monodromy matrix $h_{i}$. Then the dual gauge group $\tilde{G}$ is,

$$
\tilde{G}=S U(N)_{0} \times S U\left(n_{1} N+M_{1}^{\prime}\right)_{1} \times \ldots \times S U\left(n_{k} N+M_{k}^{\prime}\right)_{k}
$$

where

$M_{\alpha}^{\prime}=M_{\beta}\left(h_{i}\right)_{\beta \alpha}$, and $\left(h_{i}\right)_{\beta \alpha}: \beta \alpha$ component of i-th monodromy matrix. The dual matters arise as hypermutiplets in the bi-fundamental representations $\left(n_{i} N+M_{i}^{\prime}, \overline{n_{j} N+M_{j}^{\prime}}\right)$ if $\mathrm{i}$-th and $\mathrm{j}$-th gauge group factors are adjacent in the sense that the nodes are connected by spokes in the corresponding extended Dynkin diagram.

The geometrization of Seiberg's duality given above provides us with a deep insight in properties of the duality. We find a new structure in a sequence of Seiberg's dualities, i.e. the "braid group" structure. It is known in mathematics[12] and confirmed easily in simple examples, that the monodromy matrices $h_{i}$ satisfy the following relations;

$$
\begin{gathered}
h_{i+1} h_{i} h_{i+1}=h_{i} h_{i+1} h_{i} \\
h_{i} h_{j}=h_{j} h_{i}
\end{gathered}
$$

for

$$
|i-j| \geq 2
$$

This is the algebra of "braid group" with k strands. This fact combined with the relation between Seiberg's duality and Picard-Lefschetz monodromy, shows that a sequence of Seiberg's dualities taken on the gauge group factors of the product group must have the same "braid group" structure. In particular, we find from the relation of the braid algebra that successive Seiberg's duality transformations performed on an adjacent gauge group factors do not commute, whereas those on non-adjacent group factors commute. For example, let the gauge group of the original theory be;

$$
S U(N)_{0} \times S U\left(N+M_{1}\right)_{1} \times S U\left(N+M_{2}\right)_{2} \times S U\left(N+M_{3}\right)_{3} .
$$

From the braid algebra, it follows that the Seiberg's duality performed on the group factor $S U\left(N+M_{1}\right)_{1}$ first and then on $S U\left(N+M_{2}\right)_{2}$, and the Seiberg's duality performed in the reversed order, i.e. on the gauge group factor $S U\left(N+M_{2}\right)_{2}$ first and then on $S U\left(N+M_{1}\right)_{1}$, give different theories. Because, in the former case, the resulting group is 


$$
S U(N)_{0} \times S U\left(N+M_{1}^{\prime}\right)_{1} \times S U\left(N+M_{2}^{\prime}\right)_{2} \times S U\left(N+M_{3}^{\prime}\right)_{3}
$$

with,

$$
M_{i}^{\prime}=M_{k}\left(h_{1}\right)_{k j}\left(h_{2}\right)_{j i}
$$

whereas in the latter case, the resulting group is,

$$
M_{i}^{\prime}=M_{k}\left(h_{2}\right)_{k j}\left(h_{1}\right)_{j i} \text {. }
$$

The resulting groups are different in two cases since $h_{1} h_{2} \neq h_{2} h_{1}$

However, if we perform Seiberg's duality transformation three times succesively, in the distinct two ways given below, we reach the same theory.

1. Perform Seiberg's duality on $S U\left(N+M_{1}\right)_{1}$ first, then on $S U\left(N+M_{2}\right)_{2}$ and finally on $S U\left(N+M_{1}\right)_{1}$.

2. Perform Seiberg's duality on $S U\left(N+M_{2}\right)_{2}$ first, then on $S U\left(N+M_{1}\right)_{1}$ and finally on $S U\left(N+M_{2}\right)_{2}$.

This fact follows from the relation,

$$
h_{1} h_{2} h_{1}=h_{2} h_{1} h_{2}
$$

In conclusion, we have geometrized dualities between $\mathrm{N}=1$ supersymmetric gauge theories in four dimensions (Seiberg's duality), by means of AdS/CFT correspondence. It turned out that Seiberg's duality can be interpreted as a geometrical operation on the conifold, called Picard-Lefschetz monodromy. The homology 2-cycles of the conifold are transformed by this monodromy operation. If D5 branes wrap around these 2-cycles, and have D3 brane charges corresponding to the wrapping numbers, the effective D3 brane numbers are changed, by the monodromy transformation. In the field theory side, this implies that the number of colors changes upon Seiberg's duality transformation and it turned out that the rule $\tilde{N}_{c}=N_{f}-N_{c}, \tilde{N}_{f}=N_{f}$ is satisfied. Furthermore, we obtained a general expression for the dual gauge group, when Seiberg's duality is taken on one of the gauge group factor of the product gauge group of the $\mathrm{N}=1$ supersymmetric gauge theory arising from the field theory on D3 branes on generalized conifold singularity of ADE type. Moreover, we found a braid group structure in a sequence of Seiberg's dualities taken on the gauge group factors of the product gauge group, by virtue of the geometrization of Seiberg's duality in the framework of AdS/CFT correspondences. 


\section{References}

[1]J. Maldacena, Adv. Theor. Math. Phys, 2(1998)231

[2]I. R. Klebanov and E. Witten, hep-th/9807080

[3] D. R. Morrison and M. R. Plesser, hep-th/9810201

[4]S. Gubser, N. Nekrasov and S. Shatashvili, hep-th/9811230]

[5]E. Lopez, hepth/981202

[6]N. Seiberg, Nucl. Phys. B 435(1995)129

[7]Kei Ito, hep-th/9712225

[8]A. M. Uranga, hep-th/9811004

[9]K. Dasgupta and S. Mukhi, hep-th/9811139

[10]R. von Unge, hep-th/9901091

[11]S. Elitzur, A. Giveon and D. Kutasov, Phys. Lett. B 400(1997)269

[12]V. I. Arnold, S. M. Gusein-Zade and A. N. Varchenko, Singularities of Differential Maps" vol I, II. Birkhäuser, 1988, Boston, Basel, Berlin.

[13]Kei Ito, Phys. Lett. B 229(1989)379

[14]D-E. Diaconescu, M. R. Douglas and J. Gomis, hep-th/9712230 